\title{
Streptococcus gallolyticus meningocephalitis in adults: the first case report in China
}

\author{
Yue-Man Dai', Min Zhao' ${ }^{2}$, Hong-Ji Lu ${ }^{2}$, Li-Xin Wang ${ }^{2}$ \\ 'Department of Neurology, The 2nd Teaching Hospital of Guangzhou University of Chinese Medicine, Guangzhou 510120, Guangdong, \\ China. \\ ${ }^{2}$ Department of Neurology, Guangdong Provincial Hospital of Chinese Medicine, Guangzhou 510405, Guangdong, China.
}

Correspondence to: Dr. Li-Xin Wang, Department of Neurology, Guangdong Provincial Hospital of Chinese Medicine, 111 Dade Road, Guangzhou 510405, Guangdong, China. E-mail: plawlx@126.com

How to cite this article: Dai YM, Zhao M, Lu HJ, Wang LX. Streptococcus gallolyticus meningocephalitis in adults: the first case report in China. Neuroimmuno/ Neuroinflammation 2018;5:28. http://dx.doi.org/10.20517/2347-8659.2018.09

Received: 13 Mar 2018 First Decision: 28 Apr 2018 Revised: 18 May 2018 Accepted: 20 May 2018 Published: 16 Jul 2018

Science Editor: Athanassios P. Kyritsis Copy Editor: Jun-Yao Li Production Editor: Huan-Liang Wu

\begin{abstract}
We report on the first case of a Streptococcus gallolyticus meningocephalitis in China. The bacterium was first isolated from the patient's cerebrospinal fluid and has so far not been associated with human infections of the central nervous system. We hope our case report can give some references for the diagnosis and treatment of the Streptococcus gallolyticus meningocephalitis in China.
\end{abstract}

Keywords: Streptococcus gallolyticus, meningocephalitis, China, case report, adult

\section{INTRODUCTION}

Streptococcus gallolyticus is an uncommon cause of meningocephalitis so far apart from the bacteremia or the peritonitis. We report one case with meningocephalitis in which Streptococcus gallolyticus was isolated from the cerebrospinal fluid (CSF). The organisms were seen on a gram-stained preparation of CSF. The patient had a history of adenocarcinoma of colon and presented with hyperpyrexia, deliration and neck rigidity. After the antibiotic treatment for 2 weeks, finally he got fully recovery. A review of the literature revealed only about 42 cases of meningitis due to Streptococcus gallolyticus were reported and the present case report was the first one from china. The case indicates the importance of laboratory identification of specific organisms and provides experience in meningocephalitis caused by Streptococcus gallolyticus.

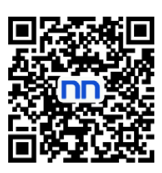


Table 1. Clinical characteristics for the patient with Streptococcus gallolyticus meningocephalitis

\begin{tabular}{ll}
\hline Characteristics & Patient data \\
\hline Age (years) & 80 \\
Gender & Male \\
Predisposing factor (s) & Adenocarcinoma of colon \\
& Bibulosity \\
Clinical presentation & \\
Temperature $\left({ }^{\circ} \mathrm{C}\right.$ ) & 39.3 \\
Neck rigidity & Yes \\
Headache & No \\
Score on Glasgow Coma Scale & 13 \\
Neurological deficits & Delirium \\
CSF findings & \\
Leukocyte count & $74 \times 10^{6} / \mathrm{L}$ \\
Protein level (mg/L) & 6058 \\
CSF/blood glucose ratio & $<0.13$ \\
Cranial CT (MRI) & Normal \\
Cultures & \\
Blood culture & Negative \\
CSF culture & Positive \\
S. gallolyticus-associated disease & \\
Endocarditis & No \\
Colon adenocarcinoma & Yes \\
Strongyloidiasis & Unknown \\
Empirical treatment & \\
Antibiotics & Meropenem, linezolid \\
Dexamethasone & No \\
Outcome & Recovery \\
\hline & \\
\hline
\end{tabular}

CSF: cerebrospinal fluid; CT: computed tomography; MRI: magnetic resonance imaging

\section{CASE REPORT}

An 80-year-old man was admitted to the emergency room of Guangdong Provincial Hospital of Chinese Medicine. He presented symptoms with deliration and neck rigidity. According to his wife's statement, the patient complained the abdominal pain 5 days ago. Meanwhile, he had a history of radical operation for adenocarcinoma of stomach and colon, as well as the percutaneous coronary intervention. Further questioning for his wife and daughter revealed that the patient has the habit of drink Chinese liquor for about $50 \mathrm{~mL}$ every day for decades before the partial resection of stomach. Even in the latest years, he still drank about $20 \mathrm{~mL}$ every day. The features of the patient are summarized in Table 1.

On examination, he was delirious with neck rigidity, but no febrile. There was no other neurological abnormalities. Meanwhile, there is no abdominal pain. Peripheral blood samples, including cultures, were taken and treatment with ceftriaxone ( $2 \mathrm{~g}$ intravenous drip, ivd q12h) as well as acyclovir (75 $\mathrm{mg}$ peros, po bid) were started immediately. Urgent brain computed tomography (CT) scanning was normal. Then the lumbar puncture was performed. Laboratory studies of CSF disclosed the following values: the CSF pressure, $130 \mathrm{cmH}_{2} \mathrm{O}$; WBCs, $15 \times 10^{6} / \mathrm{L}$; glucose < $1.11 \mathrm{mmol} / \mathrm{L}$ (serum glucose: $8.96 \mathrm{mmol} / \mathrm{L}$ ); and protein, $4946 \mathrm{mg} / \mathrm{L}$. He was diagnosed with central nervous system (CNS) infection and was transferred to the neurological department.

Day 1 physical examination showed a poor-nourished man in a mild altered consciousness. His vitals were: temperature $36.4{ }^{\circ} \mathrm{C}$, blood pressure $145 / 59 \mathrm{mmHg}$, pulse rate 66 and respiration rate 20. Neurological examination revealed a delirious man who had decreased range of motion of his neck. No other abnormalities of neurologic system as well as the respiratory and digestive system were detected. Then, blood routine, high-sensitivity C-reactive protein (hs-CRP), procalcitonin including cultures were taken again. Magnetic resonance imaging (MRI), electroencephalogram (EEG) monitoring was performed. Combining 


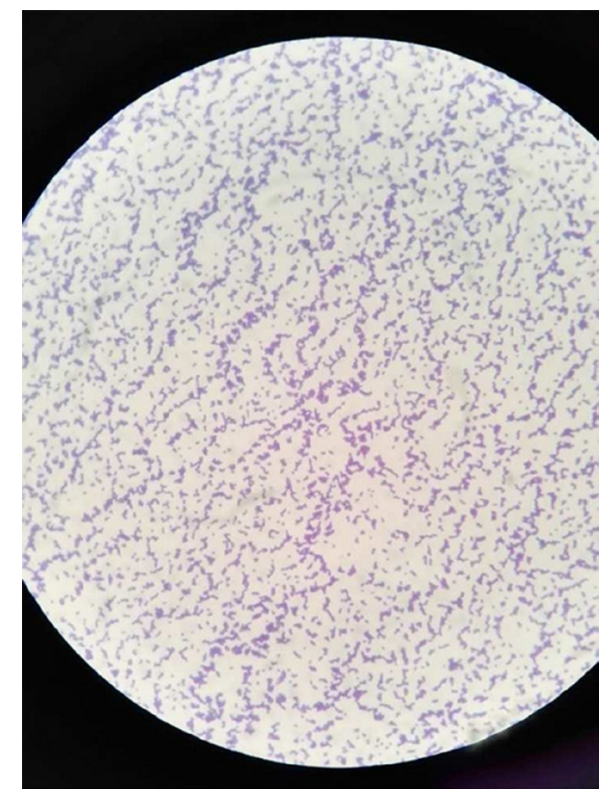

Figure 1. The microscopic view of cerebrospinal fluid culture (1:1000)

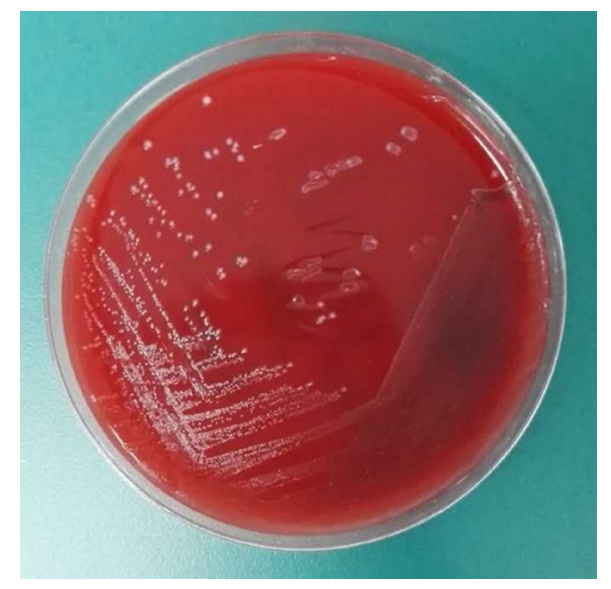

Figure 2. The macroscopic view of Streptococcus gallolyticus (1:1)

the patient's symptoms, positive signs, results of the blood test and CSF test, we considered the patient suffered from meningocephalitis and the treatment with meropenem ( $2 \mathrm{~g}$ ivd $\mathrm{q} 8 \mathrm{~h}$ ) was started. Meantime, a lumbar puncture was performed for the second time.

Blood routine showed the following values: WBCs, $12.1 \times 10^{9} / \mathrm{L}(87.9 \%$ neutrophils, $7.7 \%$ lymphocytes, $4 \%$ monocytes). And CSF test found the following values: CSF pressure, $110 \mathrm{cmH}_{2} \mathrm{O}$; WBCs, $74 \times 10^{6} / \mathrm{L}(15 \%$ neutrophils, $80 \%$ lymphocytes, $5 \%$ monocytes); glucose < $1.11 \mathrm{mmol} / \mathrm{L}$ (serum glucose: $8.33 \mathrm{mmol} / \mathrm{L}$ ); and protein, $6058 \mathrm{mg} / \mathrm{L}$. At this time, Streptococcus gallolyticus was isolated from CSF cultures [Figures 1 and 2]. Considering the permeability of linezolid through the blood brain barrier (BBB) and the sensitivity of linezolid (minimum inhibitory concentration, MIC / kindy-bauer, KB $29 \mathrm{~mm}$ ) to the Streptococcus gallolyticus, the therapy with linezolid (0.6 g ivd q12h) was started.

Brain contrast enhancement MRI showed no signs of infection and the long-term EEG monitoring found no epileptic signs. The detailed report showed the background activity was no alpha wave and diffused slow 
Table 2. Comparison of the clinical characteristic and test results with the patient

\begin{tabular}{|c|c|c|c|c|c|}
\hline Characteristics & Day1 & Day 3 & Day 8 & Day 14 & Day 28 \\
\hline \multicolumn{6}{|l|}{ Clinical presentation } \\
\hline Consciousness & Deliration & Consciousness & Consciousness & Consciousness & Consciousness \\
\hline Neck rigidity & Yes & Yes & No & No & No \\
\hline Headache & No & No & No & No & No \\
\hline Fever & No & Yes & Yes & Yes & Unknown \\
\hline Score on Glasgow Coma Scale & 13 & 13 & 15 & 15 & 15 \\
\hline Temperature $\left({ }^{\circ} \mathrm{C}\right)$ & 36.4 & 37.8 & 38.3 & 38.2 & Unknown \\
\hline \multicolumn{6}{|l|}{ CSF findings } \\
\hline Leukocyte $\left(\times 10^{6} / \mathrm{L}\right)$ & 74 & 640 & 384 & Unknown & 32 \\
\hline Protein level (mg/L) & 6058 & 5160 & 2342 & Unknown & 359 \\
\hline CSF glucose $(\mathrm{mmol} / \mathrm{L})$ & $<1.11$ & 1.47 & 1.81 & Unknown & Unknown \\
\hline \multicolumn{6}{|l|}{ Blood findings } \\
\hline Leukocyte $\left(\times 10^{9} / \mathrm{L}\right)$ & 12.1 & 19.16 & 21.89 & 11.25 & 11.16 \\
\hline Neutrophils (\%) & 87.9 & 81.2 & 87.2 & 75 & 79 \\
\hline Monocytes (\%) & 4 & 6.7 & 4.8 & 10.8 & 7.1 \\
\hline Lymphomonocyte (\%) & 7.7 & 12 & 6.7 & 11.9 & 11.9 \\
\hline Serum glucose (mmol/L) & 8.33 & 10.95 & 6.83 & 6.61 & Unknown \\
\hline \multicolumn{6}{|l|}{ Cultures } \\
\hline Blood culture & Negative & Negative & Negative & Negative & Unknown \\
\hline CSF culture & Positive & Negative & Negative & Negative & Negative \\
\hline hs-CRP (mg/L) & Unknown & Unknown & 57.04 & 34.03 & $25.3(\mathrm{CRP})$ \\
\hline Procalcitonin (ng/mL) & Unknown & 0.58 & 0.17 & 0.17 & 0.04 \\
\hline
\end{tabular}

CSF: cerebrospinal fluid; hs-CRP: high-sensitivity C-reactive protein

waves as well as low amplitude of beta were observed in all leads. No sleep cycle wave was observed as well.

Day 3, he developed a mild fever with the temperature of $37.8{ }^{\circ} \mathrm{C}$. He became conscious but still cannot communicate with the others. A lumbar puncture was performed to evaluate the effect of the therapy. The WBCs in blood and CSF increased while the glucose and protein levels were improved [Table 2]. The cultures of CSF and blood were negative.

Day 8, the physical examination showed the neck rigidity was better than before and there were few rales in both lower lungs. Deep sputum cultures yielded multidrug-resistant baumanii and the amikacin (0.2 g ivd q12h) was added.

As the neurological function was improved, we performed another lumbar puncture and the results showed the WBCs decreased to $384 \times 10^{6} / \mathrm{L}$ and protein as well as the glucose increased to $2342 \mathrm{mg} / \mathrm{L}$ and $1.81 \mathrm{mmol} / \mathrm{L}$ separately indicating the antibiotic therapy was effective. Controversially, the WBCs and hs-CRP in the blood increased. Considering the symptoms and the results of CSF tests were both improved, the therapy with meropenem and linezolid was continued.

While the treatment was effective based on the CSF results. We did the other tests to rule out the infection of the other systems and the cacotrophy conditions. An echocardioram revealed no evidence of endocarditis and colonoscopy revealed the ascending colon polyps and haemorrhoids. The chest X-ray found no tumor. The abdominal CT scan was also negative.

The results of the marker for the systemic inflammation, CSF, clinical presentation are summarized in Table 2. All together, meropenem ( $2 \mathrm{~g}$ ivd q8h) was given for 16 days, linezolid ( $0.6 \mathrm{~g}$ ivd $\mathrm{q} 12 \mathrm{~h}$ ) was given for 15 days and amikacin ( $0.2 \mathrm{~g}$ ivd $\mathrm{q} 12 \mathrm{~h}$ ) was given for 5 days. Other treatments including nutritional supporting, early rehabilitation, bronchofiberscope, bed sore prevention were also given to the patients. Finally, the patient was completely awake and transferred to the rehabilitation facility. 
Two weeks after discharge, we followed up the patient by telephone. His wife told us the lumbar puncture was performed once more in the rehabilitation facility and he could take care of himself independently. The laboratory studies of CSF and blood was almost normal [Table 2].

\section{DISCUSSION}

There are only about 42 adult cases about Streptococcus gallolyticus meningitis reported according to the literature. For now, the present case report was the first from the Chinese population. The detail of the diagnosis and treatment was documented which could provide experience for the subsequent patients of the CNS infection with Streptococcus gallolyticus.

We think that the gastrointestinal tract was probably the source of Streptococcus gallolyticus in our patient who had prior gastroenteritis and had the history of moderately differentiated adenocarcinoma of stomach treated with partial resection together with colonic adenocarcinoma. Many studies have confirmed the fact that patients with both benign and malignant of gastrointestinal lesions were susceptible to Streptococcus bovis (S. bovis) bacteremia ${ }^{[1-3]}$. A review of 119 cases of $S$. bovis endocarditis or bacteremia suggested that there were 48 patients accompanied with gastrointestinal neoplasms, 22 of which were adenocarcinoma ${ }^{[4]}$. Colonic carcinoma has been reported in up to $50 \%$ of patients with $S$. bovis bacteremia or endocarditis ${ }^{[5]}$. So, there is a strong link between Streptococcus gallolyticus infection and bowel disease. Nevertheless, the extent, nature, and basis of this association are still not completely understood.

According to the literature, the susceptible risk factor for the Streptococcus gallolyticus meningitis was cacotrophy, immunosuppression, endocarditis, colon carcinoma, strongyloidiasis and bibulosity ${ }^{[6-10]}$. Among the 42 adult patients reported ${ }^{[11]}, 43 \%$ (18/42) of the patients had the conditions such as immunosuppression, cancer and alcoholism. Meantime, 33\% (14/42) of the patients had the infection with the strongyloidiasis, $63 \%(15 / 24)$ had the colon abnormalities, $8 \%(5 / 28)$ had the endocarditis. Furthermore, endocarditis has been reported to be caused by S. gallolyticus ssp. gallolyticus more frequently than by S. gallolyticus ssp. Pasteurianus ${ }^{[12,13]}$. So, it is necessary to test for strongyloidiasis and do the echocardiography and colonoscopy for the patients with Streptococcus gallolyticus meningitis. For our patient, it is definitely that he has the cacotrophy, colon carcinoma, but not the endocarditis. Unfortunately, in our patient, the stool examination was not performed repeatedly to detect strongyloidiasis.

Even though, the CNS infection has clinical characteristics, such as ardent fever, headache and neck rigidity. In many patients with CNS infection due to Streptococcus gallolyticus, the presenting differentiated from each other, as occurred in our patient. It was not until days after admission that our patient developed the febrile signs. The delayed fever may be the reason of his advanced age, the condition of cacotrophy or the nosocomial infection.

As we all know, bacterial infection of the CNS has the following characteristics of laboratory examination: the CSF pressure, WBCs, NEUT\% and the protein were high, while the glucose in CSF was reduced. In our patient, we performed lumbar puncture repeatedly, but the CSF pressure was never higher than normal. This may be due to differences in individual immune responses. IgG and IgA but no IgM are seen in normal CSF because IgM has a larger molecular weight. Humoral immune responses often form antigen-antibody complexes; this reaction is often carried out in blood vessels, leading to severe vasculitis reactions in or near nerve tissue. It may be the characteristic for Streptococcus gallolyticus meningocephalitis which needs more clinical data. Meanwhile, we cannot ignore the fact that the cultures plays an important role for the differentiation of CNS infections. For the 42 patients, the positive incidence was $88 \%$ for CSF cultures and $87 \%$ for blood cultures ${ }^{[11]}$. We performed the blood culture repeatedly, the results were all negative. So we think that our patient might not be infected through blood but through the gastrointestinal tract. 
Now, Streptococcus gallolyticus performed as a member of S. bovis, which has three hypotypes. Before then, S. bovis strains were divided into biotypes based on their ability to decompose the mannitol (biotype I) or not (biotype II $)^{[14]}$. Biotype II was further subdivided into biotypes II.1 and II.2. Depending on the ability to produce acid from trehalose, to exhibit frequently b-glucuronidase, to degrade starch and b-galactosidase activity, Biotype II. 2 strains are distinguished from biotype II.1 strains. In 1990, Osawa ${ }^{[15]}$ suggested a new species, S. gallolyticus, isolated from fecal excretion of a koala, for those organisms able to decarboxylate gallic acid. Subsequently, the further studies suggested that the S. gallolyticus species comprised S. bovis biotypes I and II/ $2^{[16]}$. Later studies about the sequencing of soda and DNA-DNA hybridization confirmed the need for the taxonomic chang ${ }^{[17,18]}$. Therefore, Abdulamir et al ${ }^{[6]}$ suggested that the S. gallolyticus species includes three subspecies: S. gallolyticus subsp. gallolyticus, S. gallolyticus subsp. pasteurianus, and S. gallolyticus subsp. macedonicu. Among the three biotypes of Streptococcus gallolyticus, we know that $S$. gallolyticus subsp. pasteurianus causes meningitis, bacteremia, peritonitis, and chorioamnionitis in adults ${ }^{[19-21]}$. Since this was the first case of Streptococcus gallolyticus among Chinese population, we didn't detect the subtype because the technical reasons. We hope that our case report can provide some information for the detection of the Streptococcus gallolyticus related diseases and to make more precision diagnosis by the subtype detection.

From the review of the Streptococcus bovis infection of the CNS, we know that most cases of S. bovis infection can be treated with penicillin alone ${ }^{[22]}$. But as is known to all, the cultures plays an important role in the course of treatment. Savitch et al. ${ }^{[23]}$ found that patients with $S$. bovis endocarditis were resistant to penicillin G. Several researches proposed that the empirical antibiotics should be chosen based on patient history, results of CSF gram stain and local community antibiotic resistance patterns ${ }^{[24]}$. For our patient, considering the history of the patient as well as the permeability of BBB to antibiotics, we chosen the meropenem ( $2 \mathrm{~g}$ ivd q8h) firstly ${ }^{[25]}$. We added linezolid as soon as the Streptococcus gallolyticus reported. Considering the results of sputum Gram stain we continued the treatment with meropenem. Eventually, our patient recovered very well as we follow up by telephone two weeks after the discharge.

The studies suggested that the mortality rate of the Streptococcus gallolyticus meningitis patients is about $24 \%{ }^{[11]}$, but we cannot ignore the fact that the total number of reported patients with Streptococcus gallolyticus meningitis was small. So, it is of great significance to form a standardized and effective diagnosis and treatment program for Streptococcus gallolyticus meningitis.

For now, all of the reports are from European and American countries. There are no reports from China. We hope our case report can give some references for the diagnosis and treatment of the Streptococcus gallolyticus meningocephalitis in China.

\section{DECLARATIONS}

\section{Authors' contributions}

Drafting the manuscript, and literature review: Dai YM, Zhao M, Lu HJ

Revising the manuscript: Wang LX

\section{Availability of data and materials}

The data and material used in the study could be open upon request.

\section{Financial support and sponsorship}

The study was supported by China Postdoctoral Science Foundation (grant No. 2016M592513).

\section{Conflicts of interest}

All authors declared that there are no conflicts of interest relevant to this article. 


\section{Ethical approval and consent to participate}

The study is approved and the patient consent is obtained.

\section{Consent for publication}

Not applicable.

\section{Copyright}

(c) The Author(s) 2018.

\section{REFERENCES}

1. Friedrich IA, Wormser GP, Gottfried EB. The association of recent Streptococcus bovis bacteremia with colonic neoplasia. Mil Med 1982;147:584-5.

2. Reynolds JG, Silva E, McCormack WM. Association of Streptococcus bovis bacteremia with bowel disease. J Clin Microbiol 1983;17:6967.

3. Beeching NJ, Christmas TI, Ellis-Pegler RB, Nicholson GI. Streptococcus bovis bacteraemia requires rigorous exclusion of colonic neoplasia and endocarditis. Q J Med 1985;56:439-50.

4. Weitberg AB, Annese C, Ginsberg MB. Streptococcus bovis meningitis and carcinoma of the colon. Johns Hopkins Med J 1981;148:260-1.

5. Alazmi W, Bustamante M, O'Loughlin C, Gonzalez J, Raskin JB. The association of Streptococcus bovis bacteremia and gastrointestinal diseases: a retrospective analysis. Dig Dis Sci 2006;51:732-6.

6. Abdulamir AS, Hafidh RR, Abu Bakar F. The association of Streptococcus bovis/gallolyticus with colorectal tumors: the nature and the underlying mechanisms of its etiological role. J Exp Clin Cancer Res 2011;30:11.

7. Sasaki Y, Taniguchi T, Kinjo M, McGill RL, McGill AT, Tsuha S, Shiiki S. Meningitis associated with strongyloidiasis in an area endemic for strongyloidiasis and human T-lymphotropic virus-1: a single-center experience in Japan between 1990 and 2010. Infection 2013;41:1189-93.

8. Shields AM, Goderya R, Atta M, Sinha P. Strongyloides stercoralis hyperinfection presenting as subacute small bowel obstruction following immunosuppressive chemotherapy for multiple myeloma. BMJ Case Rep 2014;2014:bcr2013202234.

9. Mello R, da Silva Santos M, Golebiosvki W, Weksler C, Lamas C. Streptococcus bovis endocarditis: analysis of cases between 2005 and 2014. Braz J Infect Dis 2015;19:209-12.

10. Shimasaki T, Chung H, Shiiki S. Five cases of recurrent meningitis associated with chronic strongyloidiasis. Am J Trop Med Hyg 2015;92:601-4.

11. van Samkar A, Brouwer MC, Pannekoek Y, van der Ende A, van de Beek D. Streptococcus gallolyticus meningitis in adults: report of five cases and review of the literature. Clin Microbiol Infect 2015;21:1077-83.

12. Corredoira J, Alonso MP, Coira A, Casariego E, Arias C, Alonso D, Pita J, Rodriguez A, Lopez MJ, Varela J. Characteristics of Streptococcus bovis endocarditis and its differences with Streptococcus viridans endocarditis. Eur J Clin Microbiol Infect Dis 2008;27:28591.

13. Boleij A, van Gelder MM, Swinkels DW, Tjalsma H. Clinical Importance of Streptococcus gallolyticus infection among colorectal cancer patients: systematic review and meta-analysis. Clin Infect Dis 2011;53:870-8.

14. Parker MT, Ball LC. Streptococci and aerococci associated with systemic infection in man. J Med Microbiol 1976;9:275-302.

15. Osawa R. Formation of a clear zone on tannin-treated brain heart infusion agar by a Streptococcus sp. isolated from feces of koalas. Appl Environ Microbiol 1990;56:829-31.

16. Devriese LA, Vandamme P, Pot B, Vanrobaeys M, Kersters K, Haesebrouck F. Differentiation between Streptococcus gallolyticus strains of human clinical and veterinary origins and Streptococcus bovis strains from the intestinal tracts of ruminants. J Clin Microbiol 1998;36:35203 .

17. Poyart C, Quesne G, Trieu-Cuot P. Taxonomic dissection of the Streptococcus bovis group by analysis of manganese-dependent superoxide dismutase gene (sodA) sequences: reclassification of 'Streptococcus infantarius subsp. coli' as Streptococcus lutetiensis sp. nov. and of Streptococcus bovis biotype 11.2 as Streptococcus pasteurianus sp. nov. Int J Syst Evol Microbiol 2002;52:1247-55.

18. Schlegel L, Grimont F, Ageron E, Grimont PA, Bouvet A. Reappraisal of the taxonomy of the Streptococcus bovis/Streptococcus equinus complex and related species: description of Streptococcus gallolyticus subsp. gallolyticus subsp. nov., S. gallolyticus subsp. macedonicus subsp. nov. and S. gallolyticus subsp. pasteurianus subsp. nov. Int J Syst Evol Microbiol 2003;53:631-45.

19. Coret Ferrer F, Vilchez Padilla JJ, Igual Adell R, Ferrando Ginestar J. Streptococcus bovis meningitis: no association with colonic malignancy. Clin Infect Dis 1993;17:527-8.

20. Beck M, Frodl R, Funke G. Comprehensive study of strains previously designated Streptococcus bovis consecutively isolated from human blood cultures and emended description of Streptococcus gallolyticus and Streptococcus infantarius subsp. coli. J Clin Microbiol 2008;46:2966-72.

21. Akahane T, Takahashi K, Matsumoto T, Kawakami Y. A case of peritonitis due to Streptococcus gallolyticus subsp. pasteurianus. Kansenshogaku Zasshi 2009;83:56-9. (in Japanese)

22. Cohen LF, Dunbar SA, Sirbasku DM, Clarridge JE 3rd. Streptococcus bovis infection of the central nervous system: report of two cases and review. Clin Infect Dis 1997;25:819-23.

23. Savitch CB, Barry AL, Hoeprich PD. Infective endocarditis caused by Streptococcus bovis resistant to the lethal effect of penicillin G. Arch Intern Med 1978;138:931-4. 
24. Tan YC, Gill AK, Kim KS. Treatment strategies for central nervous system infections: an update. Expert Opin Pharmacother 2015;16:187203.

25. van de Beek D, Cabellos C, Dzupova O, Esposito S, Klein M, Kloek AT, Leib SL, Mourvillier B, Ostergaard C, Pagliano P, Pfister HW, Read RC, Sipahi OR, Brouwer MC; ESCMID Study Group for Infections of the Brain (ESGIB). ESCMID guideline: diagnosis and treatment of acute bacterial meningitis. Clin Microbiol Infect 2016;22 Suppl 3:S37-62. 